'Laboratorio de Infecciones

Respiratorias, Programa de Microbiología y Micología, Instituto de Ciencias Biomédicas, Facultad de Medicina Universidad de Chile. ${ }^{2}$ Servicio de Enfermedades Respiratorias, Hospital San José SSMN.

aLicenciado en Biología. Estudiante Doctorado en Farmacología,

Facultad de Ciencias Químicas y Farmacéuticas, Universidad de Chile. Becario Conicyt.

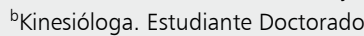
en Farmacología, Facultad de Ciencias Químicas y Farmacéuticas, Universidad de Chile. Becaria Conicyt.

Financiamiento: Proyecto Fondecyt 1100225.

Recibido el 25 de septiembre de 2012, aceptado el 2 de diciembre de 2013.

Correspondencia a:

Dr. Sergio L. Vargas Munita. Laboratorio de Infecciones Respiratorias, Programa de Microbiología y Micología, Instituto de Ciencias Biomédicas, Facultad de Medicina, Universidad de Chile Independencia 1027, Santiago, Chile. Fax: (56 2) 7325160 svargas@med.uchile.cl

\section{Dinámica y patología del barrido mucociliar como mecanismo defensivo del pulmón y alternativas farmacológicas de tratamiento}

\author{
FRANCISCO PÉREZ B. ${ }^{1, a}$, ANDREA MÉNDEZ G. ${ }^{1, b}$, \\ ANDREA LAGOS R. ${ }^{2}$, SERGIO L. VARGAS M. ${ }^{1}$
}

\section{Mucociliary clearance system in lung defense}

The first line of defense to maintain the sterility of the intrapulmonary airways is the mucociliary clearance system. This rapidly responding, nonspecific, immune defensive system is challenged by approximately 12,000 liters of air filtered by the lungs each day, containing millions of particles like spores, environmental and eventually toxic pollutants. The main components of this system are the floating mucus "blanket", the underlying mucus propelling cilia with periciliary fluid, and the airway epithelium with secretory and ciliated type of cells. The fine and coordinated regulation of these three components is critical for an effective performance. Deregulation resulting from continuous insults, inherited ion channel disease, infections or abnormal immune response may lead to mucus alterations, goblet cell hyperplasia and metaplasia, airway obstruction, air trapping and chronic lung disease. Options for treatment are limited. Understanding this system may reveal new targets for treatment of lung disease.

(Rev Med Chile 2014; 142: 606-615)

Key words: Expectorants; Lung diseases; Mucociliary clearance; Mucus.

\section{A}

1 menos 12.000 litros de aire son filtrados por los pulmones de un ser humano en un día ${ }^{1}$. Este enorme volumen contiene el oxígeno necesario para las funciones vitales, alérgenos, microorganismos, partículas y otros gases $^{2}$. Al igual que otros epitelios, el respiratorio está protegido por defensas que incluyen barreras anatómicas, cambios aerodinámicos, secreción de moco, tos, macrófagos alveolares y moléculas con efecto antibiótico ${ }^{3,4}$. La primera línea de defensa es el barrido mucociliar del epitelio respiratorio que, mediante la secreción constitutiva de moco mantiene la hidratación de la vía aérea, atrapa partículas, bacterias y virus, y contribuye sustancias antioxidantes, antiproteasas y antimicrobianas. Este barrido mucociliar elimina el moco en un proceso que exige una regulación muy fina entre el volumen y composición del moco y el volumen de líquido periciliar, de tal forma que permitan un adecuado batido ciliar ${ }^{5,6}$. Numerosos factores pueden alterar este balance al inducir sobreproducción e hipersecreción de moco o activar respuestas como la producción de sustancias antivirales, antibióticas y citoquinas proinflamatorias ${ }^{7}$. Estas respuestas pueden contribuir a la patogénesis de las enfermedades respiratorias comunes ${ }^{8}$.

Esta revisión describe la composición y funcionamiento del barrido mucociliar, sus alteraciones y terapias disponibles.

\section{Barrido mucociliar}

El barrido mucociliar requiere de equilibrio y coordinación entre sus tres componentes: la capa mucosa superficial en contacto con el lumen, capaz de adherir virtualmente cualquier partícula 
inhalada; la capa de líquido periciliar donde baten los cilios y sobre la que el moco flota; y el epitelio respiratorio subyacente, compuesto por células ciliadas y células secretoras. Los cilios propulsan la capa de moco flotante, y las células secretoras secretan moco, moléculas antimicrobianas, citoquinas y moléculas protectoras ${ }^{8,9}$. La capa de líquido periciliar en conjunto con la de moco, forman la capa de líquido superficial (LS) de la vía aérea (Figura 1$)^{3,9}$.

\section{Componentes del barrido mucociliar}

\section{Capa mucosa}

La capa mucosa actúa como barrera física y química ${ }^{3,10}$. Está constituida por más de 100 compuestos diferentes, principalmente agua (97\%) y sólidos, como proteínas, iones, lípidos y carbohidratos ${ }^{5,8}$. Su principal componente macromolecular son las mucinas, una familia de glicoproteínas de elevado peso molecular ( 2 a 20 x $10^{5} \mathrm{Da}$ ), altamente glicosiladas (50 a $90 \%$ de su masa son carbohidratos). Estas glicoproteínas son estructuralmente variadas, secretadas de manera constitutiva por las células caliciformes, y también pueden ser secretadas como respuesta a un estímulo agudo, lo que ocurre en milisegundos ${ }^{4,6,8}$. Las mucinas dotan al moco de características viscoelásticas que le permiten actuar como gel y capturar partículas inhaladas, sus cargas eléctricamente negativas generan fuerzas de repulsión contra bacterias y otros patógenos ${ }^{10-12}$. En la actualidad se han descrito 17 genes que codifican mucinas en el genoma humano, siendo MUC5AC y MUC5B las predominantes. Los genes MUC son regulados por factores de transcripción como NF-kB ${ }^{5,8,13}$.

El moco se almacena dentro de células secretoras en forma de gránulos de mucina condensada de hasta $1 \mu \mathrm{m}$ de diámetro ${ }^{4}$. Estos gránulos son rápida y violentamente exocitados en respuesta a estimulación del epitelio respiratorio y al salir de la célula expanden su volumen cientos de veces pudiendo así ocluir la vía aérea. La expansión de volumen de cada gránulo ocurre en milisegundos por la brusca repulsión electrostática de las cadenas peptídicas cargadas negativamente ${ }^{14}$. Glándulas submucosas que se encuentran bajo control nervioso también secretan moco $^{15,16}$.

\section{Capa de líquido periciliar}

La capa de líquido periciliar es un medio acuoso, de baja resistencia, ideal para el batido ciliar. Alberga moléculas con actividad antibacteriana, antifúngica y antiviral como lisozima, lactoferrina, siderocalina, lactoperoxidasa y defensinas, facilitando la acción de macrófagos epiteliales ${ }^{17,18}$. Su espesor de entre 5 y $10 \mu \mathrm{m}$ es crítico ya que permite la función de los cilios que miden 6 a $7 \mu \mathrm{m}$. Si esta capa aumenta su espesor, los extremos de los cilios no alcanzan la capa de moco y no logran su propulsión, y si disminuye, el moco se adhiere a los cilios pudiendo bloquear su movimiento ${ }^{9,19,20}$ (Figura 2).

La hidratación del LS requiere de mecanismos sofisticados de regulación, especialmente en los

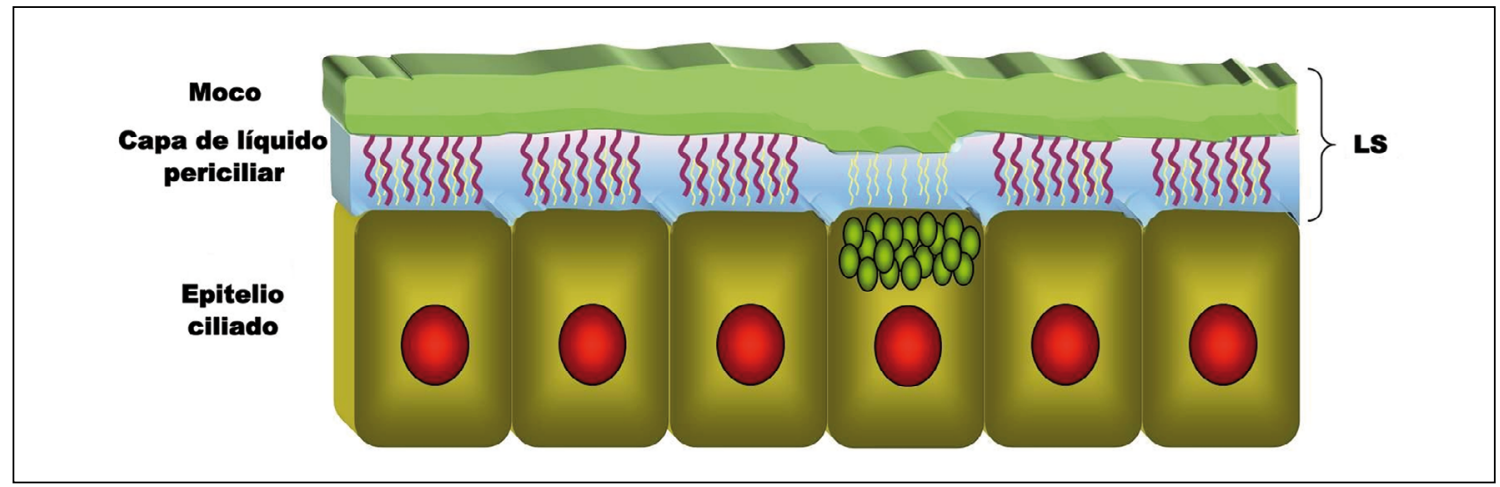

Figura 1. Componentes del barrido mucociliar: Capa superior formada por alfombra de moco, cilios inmersos en capa de líquido periciliar y células del epitelio. Se muestran células ciliadas y una célula secretora de moco (célula caliciforme) con gránulos de mucinas en su interior. Luego de ser exocitadas, las mucinas se hidratan, alcanzando volúmenes de hasta 600 veces su tamaño original; atraviesan el líquido periciliar y se unen al moco. Posteriormente, la capa mucosa es barrida fuera del aparato respiratorio. $L S=$ Líquido superficial de la vía aérea. 

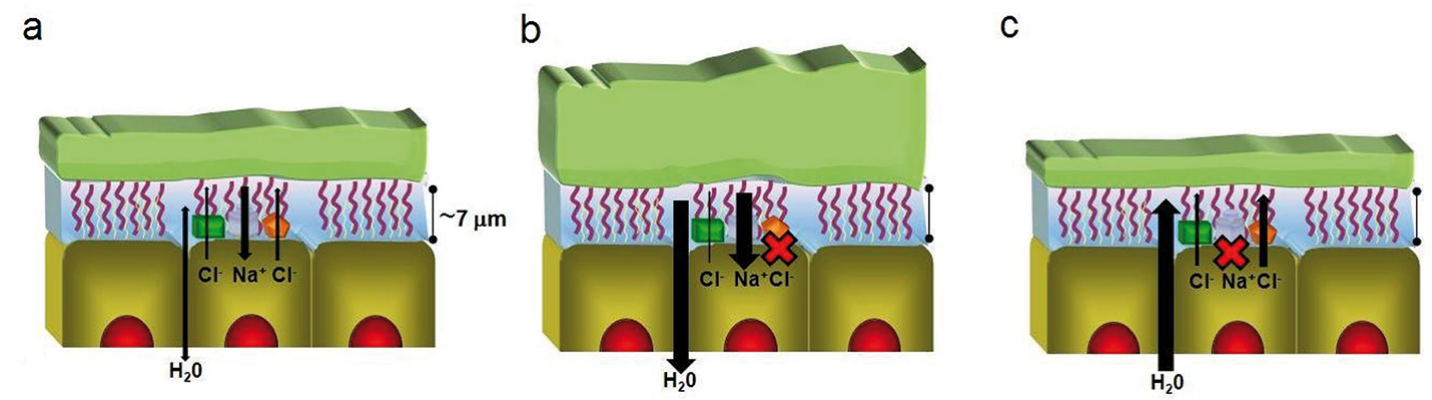

Figura 2. La hidratación del LS es el determinante principal de la eficiencia del barrido mucociliar al privilegiar la mantención del grosor $(\sim 7 \mu \mathrm{m})$ de la capa de líquido periciliar. a) En condiciones normales, existe un equilibrio dinámico entre la secreción de cloruro y la absorción de sodio. b) Ante un aumento en la cantidad de líquido (e.g. puntos de confluencia de la vía aérea, secreción de glándulas, etc.) el moco absorbe transitoriamente el exceso de agua, el canal CFTR es inhibido y el canal ENaC está activo, predominando la vía absorbente. c) Ante escasez de líquido, el moco cede agua transitoriamente, el canal ENaC es inhibido y los canales CFTR y CaCC son activados, predominando la vía secretora. CFTR = regulador de conductancia transmembrana de fibrosis quística; $\mathrm{ENaC}=$ Canal epitelial de sodio; $\mathrm{CaCC}=$ Canal de cloruro activado por calcio.

puntos de convergencia de vías aéreas. Ahí se puede generar una carga de líquido adicional, crítica, alrededor de la $3^{\text {a }}$ generación bronquial, donde consideradas globalmente las vías aéreas pequeñas suman $\sim 2 \mathrm{~m}^{2}$ de superficie y convergen en vías aéreas que suman aproximadamente $50 \mathrm{~cm}^{2}$ lo que hace en la práctica el efecto de un embudo ${ }^{21}$.

Dos mecanismos regulan el grosor del LS. El primero es la capacidad de la capa mucosa de expandirse absorbiendo y almacenando líquido, y también de contraerse para entregarlo. Normalmente el moco puede graduar en parte el espesor de la capa de líquido periciliar almacenando el exceso de líquido que se puede acumular en las zonas de confluencia de la vía aérea, y colaborando así a mantener la eficacia del batido ciliar ${ }^{9,22}$. El segundo mecanismo es la capacidad del epitelio de generar gradientes electroquímicas a través del transporte activo de iones que promueven el flujo osmótico de agua. En el epitelio respiratorio se han descrito tres canales iónicos: un canal absorbente de $\mathrm{Na}^{+}(\mathrm{ENaC})$ y dos canales secretores de $\mathrm{Cl}^{-}$ (CFTR y CaCC) (Figura 3). Ellos son modulados por moléculas como ATP, la familia de proteasas CAPs y el inhibidor de CAP. ATP aumenta LS inhibiendo ENaC e induciendo CFTR y CaCC. CAPs disminuye la hidratación de LS activando a ENaC e inhibiendo a CFTR. Probablemente la concentración de estos mediadores regula el balance entre absorción y secreción. e.g.: Al aumentar el volumen de LS se diluye ATP e inhibidores de
CAP generando una baja función de CFTR y un aumento en la actividad de ENaC. A la inversa si disminuye LS, se acumulan ATP e inhibidores de CAP activando CFTR e inactivando a $\mathrm{ENaC}^{19}$ (Figuras 2 y 3 ).

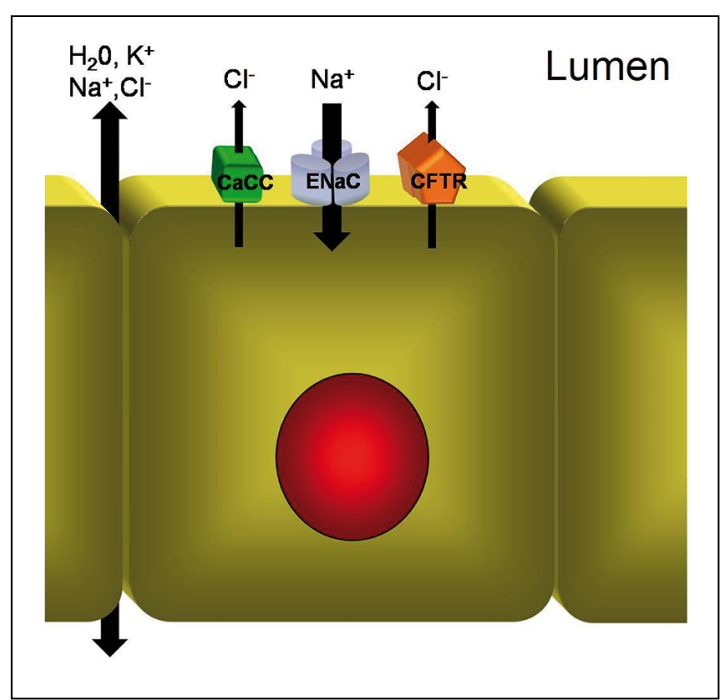

Figura 3. Regulación de la hidratación del LS. El transporte activo de iones genera gradientes electroquímicas que promueven el flujo osmótico de agua y la hidratación de mucinas exocitadas. El movimiento de agua y la mantención del equilibrio iónico son realizados por vía paracelular. ENaC = Canal epitelial de sodio; $\mathrm{CaCC}=$ Canal de cloruro activado por calcio; CFTR = Canal regulador de conductancia transmembrana de fibrosis quística. 


\section{Epitelio respiratorio}

El epitelio respiratorio remueve el moco de la vía aérea, actúa como barrera y secreta moléculas que regulan la inmunidad innata y adaptativa ${ }^{5}$. Está formado por distintos tipos celulares, fundamentalmente células ciliadas y células secretoras (caliciformes) presentes en número similar y dispuestas en mosaico. Las células secretoras poseen gran plasticidad estructural, molecular, y funcional, y se clasifican por su apariencia microscópica en células clara, mucosas, y serosas ${ }^{8}$. Células ciliadas, no ciliadas, mucosas y serosas se asocian y forman glándulas submucosas en la vía aérea cartilaginosa ${ }^{9}$.

Las células ciliadas son fácilmente reconocibles por sus cilios que baten coordinadamente y generan una onda metacronal que viaja pequeñas distancias, propulsando la capa flotante de restos celulares, microorganismos, partículas inhaladas y moco a velocidades de 4 a $20 \mathrm{~mm} / \mathrm{min}$ hacia la orofaringe para su posterior deglución o expectoración ${ }^{2,12,23}$. La frecuencia basal del batido ciliar fluctúa entre 12 y $15 \mathrm{~Hz}$ en las vías aéreas mayores, modulándose por estimulación nerviosa, mecánica y hormonal, mediante señalización intracelular mediada por $\mathrm{Ca}^{+2}$ 24. A nivel traqueal, el flujo de aire estimula mecanoreceptores que liberan ATP al extracelular, movilizando iones $\mathrm{Ca}^{+2}$ hacia el intracelular vía inositol 1,4,5-trifosfato $\left(\mathrm{IP}_{3}\right)$, incrementando la frecuencia del batido ${ }^{2,25,26}$. Otras moléculas intracelulares involucradas en el control del batido ciliar son el AMP cíclico, calmodulina, $\mathrm{IP}_{3}$, y óxido nítrico ${ }^{2}$.

\section{Barrido mucociliar en enfermedades respiratorias}

El barrido efectivo del moco es esencial para mantener el pulmón sano ${ }^{8}$. Una excesiva producción de moco impide su eliminación, aumenta la resistencia al flujo aéreo obstruyendo la vía aérea, y favorece la colonización bacteriana. Trastornos en su viscoelasticidad alteran las defensas contra las infecciones, inhibiendo la migración de neutrófilos, entre otros factores ${ }^{27,28}$.

Alteraciones en el barrido participan en la patogenia de las enfermedades que se explican a continuación.

\section{Asma bronquial}

El asma es una enfermedad inflamatoria crónica que se caracteriza por hiperreactividad de la vía aérea, obstrucción por moco y cambios estructurales como proliferación de fibroblastos, músculo liso y células caliciformes. Estas alteraciones pueden perpetuarse por la acción de mediadores de inflamación ${ }^{29,30}$. La eficacia del barrido mucociliar disminuye especialmente durante exacerbaciones de la enfermedad ${ }^{31,32}$.

La excesiva secreción de moco por sobreproducción de mucinas y cambio en los patrones de expresión por hipertrofia e hiperplasia de células caliciformes es característica del asma ${ }^{4}$. También se ha descrito hipertrofia de glándulas submucosas que podrían hipersecretar o cambiar las características reológicas del moco, generando secreciones de gran viscosidad difíciles de remover, y que pueden obstruir la vía aérea ${ }^{31,32}$. Esta acumulación de moco es característica del asma fatal27,33. Pacientes con asma presentan más células, moco y más mucinas MUC5AC y MUC5B en el lumen que individuos sanos ${ }^{34,35}$.

La inflamación de la vía aérea del asma también ha sido relacionada a disfunción mucociliar ${ }^{31,36}$. Linfocitos tipo Th2 e IL-13 juegan un rol crítico en este proceso $^{37}$; la producción de mucinas inducida por IL-13 activa una cascada de señales que lleva a la producción de MUC5AC y a un incremento en el número de células caliciformes ${ }^{38}$.

\section{Enfermedad pulmonar obstructiva crónica (EPOC)}

EPOC se caracteriza por obstrucción crónica del flujo espiratorio manifestada clínicamente como bronquitis crónica con o sin enfisema, fibrosis, daño de tejido e inflamación de la vía aérea pequeña ${ }^{39}$. Su principal causa es el tabaquismo ${ }^{40}$. El tabaco altera el barrido mucociliar al disminuir la frecuencia del batido ciliar y modificar estructuralmente los cilios que son $10 \%$ más cortos que los de no fumadores ${ }^{40}$. La inflamación pulmonar en EPOC se asocia a incremento en la producción de moco y barrido mucociliar defectuoso, alterando la respuesta epitelial y permitiendo la colonización por microorganismos, favoreciendo la infección ${ }^{41,42}$.

El incremento en número de células secretoras de moco en el epitelio y de glándulas submucosas en la vía aérea es característico de $\mathrm{EPOC}^{43}$; MUC5AC y MUC5B son las mucinas poliméricas predominantes ${ }^{43-46}$. MUC5B parece ser más prominente en el esputo de pacientes con EPOC y se correlaciona con menor función pulmonar ${ }^{44}$. 


\section{Fibrosis quística (FQ)}

La FQ es la enfermedad genética autosómica recesiva letal más común en caucásicos ${ }^{47}$. El defecto primario es una alteración en el transporte de iones a través de las superficies epiteliales debido a menor expresión del canal regulador de conductancia transmembrana de fibrosis quística CFTR, transportador de $\mathrm{Cl}^{-}$. En el epitelio respiratorio esta anormalidad se manifiesta en marcada disminución de la conductancia de $\mathrm{Cl}^{-}$y aumento en la absorción de $\mathrm{Na}^{+}$, resultando un LS deshidratado ${ }^{47-50}$. El moco se torna más viscoso y se adhiere a la superficie epitelial, colapsando la capa de líquido periciliar y frenando el movimiento ciliar $^{50,51}$. Además se inactivan los compuestos con actividad antimicrobiana ${ }^{3,10}$. Así, la FQ se caracteriza por acumulación de secreciones mucosas y colonización crónica por patógenos bacterianos como Staphylococcus aureus y Pseudomona aeruginosa $^{4,47,51-53}$. La infección e inflamación crónica llevan a proliferación de células caliciformes e hipertrofia de glándulas secretoras de moco, estableciendo un círculo vicioso de infección e injuria que lleva a insuficiencia respiratoria y muerte prematura $^{47}$.

\section{Diskinesia ciliar primaria (DCP)}

La DCP es una enfermedad autosómica recesiva asociada a situs viscerum inversus [Síndrome de Kartagener] en aproximadamente la mitad de los casos. El barrido mucociliar está alterado debido a motilidad defectuosa de los cilios, llevando a cambios pulmonares, bronquiectasias, tapones mucosos y engrosamiento peribronquial ${ }^{54}$. En la mayoría de los pacientes con DCP los cilios presentan el mismo defecto ultraestructural, consistente en ausencia de brazos de dineína, esenciales para el movimiento ciliar ${ }^{55,56}$. Otros defectos son ausencia o dislocación de microtúbulos centrales y anormalidades de microtúbulos ciliares periféricos ${ }^{56}$. Estos defectos alteran el batido ciliar y explican la inflamación e infección bronquial crónica en pacientes con $\mathrm{DCP}^{57}$.

\section{Infección e inflamación}

Las infecciones son capaces de dañar el epitelio ciliado y modificar la producción de líquido periciliar y moco alterando el mecanismo de defensa mucociliar ${ }^{3}$. Infecciones pueden inducir hiperplasia y metaplasia de células caliciformes e hipertrofia de glándulas submucosas con pro- ducción copiosa de mucinas, alterando la composición del moco y el balance entre secreción y remoción de la vía aérea, generando tapones mucosos que obstruyen el paso del aire, con consecuencias clínicas como hipoxemia, hipercapnia y prolongación de la infección ${ }^{48,62}$. Patógenos, como Mycoplasma pneumoniae pueden secretar factores de virulencia capaces de cambiar la orientación de los cilios o disminuir su frecuencia de batido ${ }^{10,58,59}$. Infecciones virales pueden aumentar la expresión de ecto-ATPasas que agotan el ATP extracelular, inhibiendo la secreción de $\mathrm{Cl}^{-}$y aumentando la viscosidad del moco $^{3}$. La respuesta inmune del hospedero a infecciones inespecíficas también puede alterar el barrido mucociliar ${ }^{3,60}$. La producción de leucotrienos, citoquinas, quimioquinas y la secreción de especies reactivas de oxígeno en el sitio de infección generan inflamación y daño en el epitelio, exponiendo terminales nerviosas sensitivas a ser estimuladas más fácilmente ${ }^{9}$. La liberación de ADN de patógenos y células del hospedero también modifica las propiedades viscoelásticas del moco, inactivando sus compuestos microbicidas e interfiriendo en su movilidad ${ }^{61}$. Los mecanismos de daño de infecciones sobre el barrido mucociliar constituyen un área de creciente interés en investigación.

\section{Tratamiento}

Existen diversas clases de compuestos mucoactivos, entre los que se encuentran expectorantes, mucolíticos, mucokinéticos y mucoreguladores (Tabla 1). El mecanismo de acción para la mayor parte de estos compuestos es desconocido o está incompletamente caracterizado.

Los expectorantes facilitarían la expectoración al incrementar la hidratación del moco. También tendrían propiedades irritantes, promoviendo la tos y permitiendo la eliminación del moco. Entre los expectorantes se encuentran el ambroxol y la solución salina hipertónica (HS). Ambroxol inhibiría la producción y expresión de MUC5AC in vitro ${ }^{63}$. HS induciría la salida osmótica de agua hacia el lumen de la vía aérea, hidratando el moco y disociaría los enlaces iónicos del moco, disminuyendo su viscosidad ${ }^{64-67}$.

Los mucolíticos disminuirían la viscosidad del moco degradando los polímeros de mucinas o ADN. Entre éstos se encuentran $\mathrm{N}$-acetilcisteína y 
Tabla 1. Agentes Mucoactivos y su utilidad según patología

\begin{tabular}{|c|c|c|c|c|c|}
\hline $\begin{array}{l}\text { Agente } \\
\text { mucoactivo }\end{array}$ & $\begin{array}{l}\text { Mecanismo potencial } \\
\text { de acción }\end{array}$ & Patología & Dosis & $\begin{array}{c}\text { Vía de } \\
\text { administración }\end{array}$ & Disponibilidad \\
\hline \multicolumn{6}{|l|}{ Expectorantes } \\
\hline Manitol & $\begin{array}{l}\text { Atrae agua hacia el } \\
\text { lumen de la vía aérea }\end{array}$ & $\mathrm{FQ}$ & $\begin{array}{l}300 \mathrm{mg} / \mathrm{día} \\
\text { polvo seco }\end{array}$ & Inhalatoria & En investigación \\
\hline $\begin{array}{l}\text { Solución salina } \\
\text { hipertónica (7\%) }\end{array}$ & $\begin{array}{l}\text { Incrementa el volumen } \\
\text { de secreción y probable- } \\
\text { mente la hidratación }\end{array}$ & $\mathrm{FQ}$ & $\begin{array}{l}4 \mathrm{ml} \text { al } 7 \% \text { dos } \\
\text { veces al día }\end{array}$ & Inhalatoria & $\begin{array}{l}\text { Preparación para } \\
\text { nebulización: } \\
\text { 1,5 ml NaCl al } 20 \% \text { en } \\
3,5 \mathrm{ml} \text { agua destilada }\end{array}$ \\
\hline Ambroxol & $\begin{array}{l}\text { Podría incrementar la } \\
\text { efectividad de la tos } \\
\text { por estimulación de la } \\
\text { secreción de surfactante }\end{array}$ & $\begin{array}{l}\text { Evidencia } \\
\text { insuficiente }\end{array}$ & $\begin{array}{l}\text { Evidencia } \\
\text { insuficiente }\end{array}$ & $\begin{array}{c}\text { Oral } \\
\text { Parenteral }\end{array}$ & $\begin{array}{l}\text { Jarabe } 15 \mathrm{mg} / 5 \mathrm{ml} \mathrm{y} \\
30 \mathrm{mg} / 5 \mathrm{ml} \\
\text { Gotas } 7,5 \mathrm{mg} / 1 \mathrm{ml} \\
\text { Comprimidos } 30 \mathrm{mg} \\
\text { Ampolla } 15 \mathrm{mg} / 2 \mathrm{ml}\end{array}$ \\
\hline \multicolumn{6}{|l|}{ Mucolíticos } \\
\hline N-acetilcisteína & $\begin{array}{l}\text { Disocia enlaces disulfuro } \\
\text { Antioxidante y antiinfla- } \\
\text { matorio }\end{array}$ & $\begin{array}{l}\text { Asma y } \\
\text { EPOC }\end{array}$ & $\begin{array}{l}200 \text { mg 2-3 } \\
\text { veces/día }\end{array}$ & $\begin{array}{c}\text { Oral } \\
\text { Inhalatoria }\end{array}$ & $\begin{array}{l}\text { Jarabe } 2 \mathrm{~g} / 100 \mathrm{ml} \\
\text { Solución } 10 \mathrm{~g} / 100 \mathrm{ml} \\
\text { para nebulización }\end{array}$ \\
\hline Dornasa alfa & $\begin{array}{l}\text { Hidroliza polímeros de } \\
\text { DNA y reduce la longitud } \\
\text { del DNA }\end{array}$ & $\mathrm{FQ}$ & 2,5 mg/día & Inhalatoria & $\begin{array}{l}\text { Ampolla } 2,5 \text { mg }(2,5 \mathrm{ml}) \\
\text { para nebulización }\end{array}$ \\
\hline \multicolumn{6}{|l|}{ Mucokinéticos } \\
\hline $\begin{array}{l}\text { Broncodilatadores } \\
\beta \text { agonista }\end{array}$ & $\begin{array}{l}\text { Incrementan el flujo } \\
\text { aéreo, el batido ciliar, la } \\
\text { secreción de } \mathrm{Cl} \text { /agua y } \\
\text { secreción de mucinas }\end{array}$ & $\begin{array}{l}\text { Asma y } \\
\text { EPOC }\end{array}$ & $\begin{array}{l}\text { Salmeterol } \\
50 \mu \mathrm{g} / \mathrm{dí} a\end{array}$ & Inhalatoria & $\begin{array}{l}\text { Inhalador dosis medida } \\
25 \mu \mathrm{g}\end{array}$ \\
\hline Surfactante & $\begin{array}{l}\text { Reduce la adherencia de } \\
\text { mucus al epitelio }\end{array}$ & $\begin{array}{l}\text { Bronquitis } \\
\text { crónica }\end{array}$ & 607,5 mg/día & Inhalatoria & $\begin{array}{l}\text { No disponible como } \\
\text { mucoactivo }\end{array}$ \\
\hline \multicolumn{6}{|l|}{ Mucoreguladores } \\
\hline Glucocorticoides & $\begin{array}{l}\text { Podrían reducir el proceso } \\
\text { de hipersecreción crónica } \\
\text { de mucus por su actividad } \\
\text { antiinflamatoria }\end{array}$ & $\begin{array}{l}\text { Asma } \\
\text { Reducido } \\
\text { efecto en } \\
\text { EPOC y FQ }\end{array}$ & $\begin{array}{l}\text { Prednisona } \\
40 \mathrm{mg} / \mathrm{dí} a \\
\text { Budesonida } \\
800 \mu \mathrm{g} / \mathrm{dí} a \\
\text { Fluticasona } \\
500 / 1.000 \mu \mathrm{g} / \text { día }\end{array}$ & $\begin{array}{c}\text { Oral } \\
\text { Inhalatoria }\end{array}$ & $\begin{array}{l}\text { Prednisona } 5 \text { y } 20 \text { mg/día } \\
\text { Budesonida } 200 \mu \mathrm{g} / \text { día } \\
\text { Fluticasona } 250 \mu \mathrm{g} / \text { día }\end{array}$ \\
\hline $\begin{array}{l}\text { Antibióticos } \\
\text { macrólidos }\end{array}$ & $\begin{array}{l}\text { Podrían reducir el proceso } \\
\text { de hipersecreción crónica } \\
\text { de mucus por su actividad } \\
\text { antiinflamatoria }\end{array}$ & $\begin{array}{l}\text { Bronquitis } \\
\text { crónica }\end{array}$ & $\begin{array}{l}\text { Claritromicina } \\
400 \text { mg/día }\end{array}$ & Oral & Comprimidos 500 mg \\
\hline $\begin{array}{l}\text { Broncodilatadores } \\
\text { anticolinérgicos }\end{array}$ & $\begin{array}{l}\text { Inhiben la secreción de } \\
\text { mucus inducida por } \\
\text { estímulo colinérgico }\end{array}$ & $\begin{array}{l}\text { Asma y } \\
\text { EPOC }\end{array}$ & $\begin{array}{l}\text { Ipratropio } \\
40 \mu \mathrm{g} / \mathrm{día} \\
\text { Tiotropio } \\
18 \mu \mathrm{g} / \text { día }\end{array}$ & Inhalatoria & $\begin{array}{l}\text { Ipratropio 0,25 mg/1 ml } \\
\text { para nebulización } \\
\text { Ipratropio en inhalador } \\
\text { dosis medida } 20 \mu \mathrm{g} \\
\text { Tiotropio en polvo seco } \\
18 \mu \mathrm{g}\end{array}$ \\
\hline
\end{tabular}


desoxirribonucleasa humana recombinante (dornasa alfa o rhDNasa). $\mathrm{N}$-acetilcisteína disociaría los puentes disulfuro de las mucinas, además de tener propiedades antioxidantes. rhDNasa reduciría la viscosidad del esputo al degradar el ADN liberado desde neutrófilos necróticos ${ }^{64-66}$.

Los mucokinéticos mejorarían la transportabilidad del moco. Entre éstos se encuentran el surfactante que reduce la adherencia del moco al epitelio, y los broncodilatadores agonistas de receptores adrenérgicos $\beta_{2}$ que incrementan el barrido mucoso al aumentar el flujo aéreo y el batido ciliar ${ }^{64-66}$.

Otro grupo son los mucoreguladores, los que no tendrían una influencia directa en el moco de la vía aérea pero podrían reducir la hipersecreción crónica de moco gracias a su actividad antiinflamatoria, mecanismo que explicaría la acción de glucocorticoides y antibióticos macrólidos. Otros mucoreguladores son los broncodilatadores anticolinérgicos, que inhiben la secreción de moco inducida por estímulo nervioso colinérgico ${ }^{64-66}$.

\section{Selección del fármaco a utilizar}

Las guías clínicas no recomiendan fármacos mucoactivos en EPOC ni asma debido a que su uso no mejora significativamente la función pulmonar. En pacientes con EPOC, el uso de $600 \mathrm{mg} /$ día de $\mathrm{N}$-acetilcisteína se ha asociado a disminución en la viscosidad del esputo e incremento de la capacidad antioxidante, pero los resultados de su administración durante períodos de exacerbación y su rol en la prevención de exacerbaciones son contradictorios ${ }^{68-70}$. Un estudio con bajo número de pacientes sugiere que la administración de 607,5 $\mathrm{mg}$ /día de surfactante aerosolizado podría mejorar la transportabilidad del moco y la función pulmonar en bronquitis crónica estable ${ }^{71}$. Los broncodilatadores $\beta$ agonistas de acción corta muestran un mínimo efecto sobre el barrido mucociliar en pacientes con asma y EPOC, mientras que los de acción prolongada han demostrado ser más efectivos $^{72-74}$. Salmeterol aumenta la frecuencia del batido ciliar mejorando el barrido, aunque no tendría un efecto directo sobre las características del moco $^{74,75}$. Formoterol $24 \mu \mathrm{g}$ administrado dos veces al día reduciría la inflamación neutrofílica en asma contribuyendo a la prevención de exacerbaciones $^{74,76}$. La utilización de anticolinérgicos como ipratropio $40 \mu \mathrm{g}$ y tiotropio $18 \mu \mathrm{g}$ mejoraría el clearance mucociliar ${ }^{74,77}$.
Algunos estudios indican que rhDNasa 2,5 mg/ día mejora significativamente la función pulmonar en comparación a solución salina hipertónica 10 $\mathrm{ml}$ al $5 \%$ y $7 \%$ en pacientes con fibrosis quísti$\mathrm{ca}^{66,78,79}$. El beneficio de otras alternativas como los broncodilatadores en pacientes con FQ es controversial $^{74}$. Asimismo, el potencial mucolítico de $\mathrm{N}$-acetilcisteína en FQ no ha sido confirmado en estudios clínicos ${ }^{67}$.

Tanto la efectividad de rhDNasa nebulizada, como la de la solución salina hipertónica o la de la N-acetilcisteína, no se han demostrado en pacientes con $\mathrm{DCP}^{80}$.

\section{Conclusiones}

El mecanismo de defensa mucociliar es fundamental para la mantención de la esterilidad en la vía aérea. La correcta integración de sus componentes permite una respuesta inespecífica rápida y eficiente. Se ha descrito que MUC5B y MUC5AC son las mucinas más frecuentes presentes en el moco sano. Sin embargo, no se sabe si alteraciones en sus proporciones relativas cambia o no las propiedades del moco ${ }^{62}$. Interesantemente MUC5AC es secretada por células caliciformes y MUC5B por glándulas submucosas, lo que sugiere que el epitelio podría responder a distintas agresiones con secreciones diferentes ${ }^{81}$. Otra observación interesante es que MUC5AC es 10 veces más abundante que MUC5B en individuos sanos, lo que cambia en asma, FQ y EPOC donde predomina MUC5B ${ }^{62}$.

Las alteraciones del barrido mucociliar contribuyen a la morbilidad de enfermedades respiratorias como asma y EPOC, y pueden favorecer infecciones. Estas alteraciones también agravan enfermedades respiratorias menos frecuentes, como FQ y DCP. Esfuerzos de investigación no han logrado, hasta la fecha, encontrar un tratamiento efectivo.

\section{Referencias}

1. Rogers DF. The airway goblet cell. Int J Biochem Cell Biol 2003; 35: 1-6.

2. Thomas R. Innate Immunity in the Lungs. Proc Am Thorac Soc 2005; 2: 403-11.

3. Chilvers MA, O'callaghan C. Local mucociliary defence mechanisms. Paediatr Respir Rev 2000; 1: 27-34. 
4. Rose MC, Voynow JA. Respiratory tract mucin genes and mucin glycoproteins in health and disease. Physiol Rev 2006; 86: 245-78.

5. Vareille M, Kieninger E, Edwards MR, Regamey N. The Airway Epithelium: Soldier in the Fight against Respiratory Viruses. Clin Microbiol Rev 2011; 210-29.

6. Voynow JA, Rubin BK. Mucins, Mucus, and Sputum. Chest 2009; 135: 2.

7. Hiemstra P. Epithelial antimicrobial peptides and proteins: their role in host defence and inflammation. Paediatr Respir Rev 2001; 2: 306-10.

8. Fahy JV, Dickey BF. Airway Mucus Function and Dysfunction. N Engl J Med 2010; 363: 2233-47.

9. Randell SH, Boucher RC. Effective Mucus Clearance Is Essential for Respiratory Health. Am J Respir Cell Mol Biol 2006; 35: 20-8.

10. Boucher RC. Cystic fibrosis: a disease of vulnerability to airway surface dehydration. Trends Mol Med 2007; 13: 231-40.

11. Hattrup CL, Gendler SJ. Structure and function of the cell surface (tethered) mucins. Annu Rev Physiol 2008; 70: 431-57.

12. Thai P, Loukoianov A, Wachi S, Wu R. Regulation of airway mucin gene expression. Annu Rev Physiol 2008; 70: 405-29.

13. Rose MC, Nickola TJ, Voynow JA. Airway mucus obstruction: mucin glycoproteins, MUC gene regulation and goblet cell hyperplasia. Am J Respir Cell Mol Biol 2001; 25: 533-7.

14. Verdugo P. Mucin exocytosis. Am Rev Respir Dis 1991; 144 (3 Pt 2): S33-7.

15. Wine JJ, Joo NS. Submucosal glands and airway defense. Proc Am Thorac Soc 2004; 1: 47-53.

16. Wine JJ. Parasympathetic control of airway submucosal glands: central reflexes and the airway intrinsic nervous system. Auton Neurosci 2007; 133: 35-54.

17. Wanner A, Salathe M, O'riordan TG. Mucociliary clearance in the airways. Am J Respir Crit Care Med 1996; 154: 1868-902.

18. Travis SM, Conway BA, Zabner J, Smith JJ, Anderson NN, Singh PK, et al. Activity of abundant antimicrobials of the human airway. Am J Respir Cell Mol Biol 1999; 20: 872-9.

19. Chambers LA, Rollins BM, Tarran R. Liquid movement across the surface epithelium of large airways. Respir Physiol Neurobiol 2007; 159: 256-70.

20. Sleigh MA, Blake JR, Liron N. The propulsion of mucus by cilia. Am Rev Respir Dis 1988; 137: 726-41.

21. Boucher RC. Regulation of airway surface liquid volume by human airway epithelia. Pflugers Arch 2003; 445: 495-8.
22. Tarran R, Grubb BR, Gatzy JT, Davis CW, Boucher RC. The relative roles of passive surface forces and active ion transport in the modulation of airway surface liquid volume and composition. J Gen Physiol 2001; 118: 223-36.

23. Sackner MA, Rosen MJ, Wanner A. Estimation of tracheal mucous velocity by bronchofiberscopy. J Appl Physiol 1973; 34: 495-9.

24. Chilvers MA, O'callaghan C. Analysis of ciliary beat pattern and beat frequency using digital high speed imaging: comparison with the photomultiplier and photodiode methods. Thorax 2000; 55: 314-7.

25. Nakahari T. Regulation of ciliary beat frequency in airways: shear stress, ATP action, and its modulation. Am J Physiol Lung Cell Mol Physiol 2007; 292: 612-3.

26. Winters SL, Davis CW, Boucher RC. Mechanosensitivity of mouse tracheal ciliary beat frequency: roles for $\mathrm{Ca} 2+$, purinergic signaling, tonicity, and viscosity. Am J Physiol Lung Cell Mol Physiol 2007; 292: 614-24.

27. Bosse Y, Riesenfeld E, Pare PD, Irvin CG. It's Not All Smooth Muscle: Non-Smooth-Muscle Elements in Control of Resistance to Airflow. Annu Rev Physiol 2010; 72: 437-62.

28. Cone RA. Barrier properties of mucus. Adv Drug Deliv Rev 2009; 61: 75-85.

29. Rogers DF. Pulmonary mucus: Pediatric perspective. Pediatr Pulmonol 2003; 36: 178-88.

30. Girodet PO, Ozier A, Bara I, Tunon de Lara JM, Marthan $\mathrm{R}$, Berger P. Airway remodeling in asthma: New mechanisms and potential for pharmacological intervention. Pharmacol Ther 2011; 130 (3): 325-37.

31. Houtmeyers E, Gosselink R, Gayan-Ramírez G, Decramer M. Regulation of mucociliary clearance in health and disease. Eur Respir J 1999; 13 (5): 1177-88.

32. Hasani A, Toms N, O'connor J, Dilworth JP, Agnew JE. Efect of salmeterol xinafoate on lung mucociliary clearance in patients with asthma. Respir Med 2003; 97 : 667-71.

33. Hays SR, Fahy JV. The role of mucus in fatal asthma. Am J Med 2003; 115: 68-9.

34. Kuyper LM, Pare PD, Hogg JC, Lambert RK, Ionescu D. Characterization of airway plugging in fatal asthma. Am J Med 2003; 115: 6-11.

35. Caramori G, Di Gregorio C, Carlstedt I, Casolari P, Guzzinati I, Adcock IM, et al. Mucin expression in peripheral airways of patients with chronic obstructive pulmonary disease. Histopathology 2004; 45 (5): 477-84.

36. Sivaprasad U, Askew DJ, Ericksen M, Gibson A, Stier MT, Brandt EB, et al. A nonredundant role for mouse Serpinb3a in the induction of mucus production in asthma. J Allergy Clin Immunol 2011; 127: 254-61. 
37. Izuhara K, Ohta S, Shiraishi H, Suzuki S, Taniguchi K, Toda S, et al. The Mechanism of Mucus Production in Bronchial Asthma. Curr Med Chem 2009; 16: 2867-75.

38. Lai H, Rogers DF. New Pharmacotherapy for Airway Mucus Hypersecretion in Asthma and COPD: Targeting Intracellular Signaling Pathways. J Aerosol Med Pulm Drug Deliv 2010; 23 (4): 219-31.

39. Bhowmik A, Chahal K, Austin G, Chakravorty I. Improving mucociliary clearance in chronic obstructive pulmonary disease. Respir Med 2009; 103 (4): 496-502.

40. Leopold PL, O'mahony MJ, Lian XJ, Tilley AE, Harvey BG, Crystal RG. Smoking Is Associated with Shortened Airway Cilia. PLoS One 2009; 4 (12): e8157.

41. Hogg J. Pathophysiology of airflow limitation in chronic obstructive pulmonary disease. Lancet 2004; 364: 70921 .

42. Hogg JC, Chu F, Utokaparch S, Woods R, Elliott WM, Buzatu L, et al. The Nature of Small-Airway Obstruction in Chronic Obstructive Pulmonary Disease. N Engl J Med 2004; 350: 2645-53.

43. Leikauf GD, Borchers MT, Prows DR, Simpson LG. Mucin apoprotein expression in COPD. Chest 2002; 121: 166S-182S.

44. Kirkham S, Kolsum U, Rousseau K, Singh D, Vestbo J, Thornton DJ. MUC5B Is the Major Mucin in the Gel Phase of Sputum in Chronic Obstructive Pulmonary Disease. Am J Respir Crit Care Med 2008; 178: 1033-9.

45. Kirkham S, Sheehan JK, Knight D, Richardson PS, Thornton DJ. Heterogeneity of airways mucus: variations in the amounts and glycoforms of the major oligomeric mucins MUC5AC and MUC5B. Biochem J 2002; 361: 537-46.

46. Deshmukh H, Shaver C, Case LM, Dietsch M. AcroleinActivated Matrix Metalloproteinase 9 Contributes to Persistent Mucin Production. Am J Respir Cell Mol Biol 2008; 38: 446-54.

47. Robinson M, Bye P. Mucociliary Clearance in Cystic Fibrosis. Pediatr Pulmonol 2002; 33: 293-306.

48. Smit JJ, Lukacs NW. A closer look at chemokines and their role in asthmatic responses. Eur J Pharmacol 2006; 533: 277-88.

49. Davis PB. Cystic Fibrosis Since 1938. Am J Respir Crit Care Med 2006; 173: 475-82.

50. Tarran R, Button B, Boucher RC. Regulation of normal and cystic fibrosis airway surface liquid volume by phasic shear stress. Annu Rev Physiol 2006; 68: 543-61.

51. Widdicombe JH. Regulation of the depth and composition of airway surface liquid. J Anat 2002; 201: 313-8.

52. Rogers DF. Airway mucus hypersecretion in asthma: an undervalued pathology? Curr Opin Pharmacol 2004; 4: 241-50.
53. Gaggar A, Rowe SM, Hardision M, Blalock JE. ProlineGlycine-Proline (PGP) and High Mobility Group Box Protein-1 (HMGB1): Potential Mediators of Cystic Fibrosis Airway Inflammation. Open Respir Med J 2010; 4: 32-8.

54. Santamaria F, Montella S, Tiddens HA, Guidi G, Casotti $\mathrm{V}$, Maglione M, et al. Structural and functional lung disease in primary ciliary dyskinesia. Chest 2008; 134 (2): 351-7.

55. Escudier E, Duquesnoy P, Papon JF, Amselem S. Ciliary defects and genetics of primary ciliary dyskinesia. Paediatr Respir Rev 2009; 10: 51-4.

56. Sirvancia S, Uyanb ZS, Ercana F, Karadagb B, Ersub R, Karakocb F, et al. Quantitative analysis of ciliary ultrastructure in patients with primary ciliary dyskinesia. Acta histochem 2008; 110: 34-41.

57. Bush A, Payne D, Pike S, Jenkins G, Henke MO, Rubin BK. Mucus Properties In Children With Primary Ciliary Dyskinesia. Chest 2006; 129: 118-23.

58. Marthin JK, Mortensen J, Pressler T, Nielsen KG. Pulmonary Radioaerosol Mucociliary Clearance in Diagnosis of Primary Ciliary Dyskinesia. Chest 2007; 132: 966-76.

59. Pittet LA, Hall-Stoodley L, Rutkowski MR, Harmsen AG. Influenza Virus Infection Decreases Tracheal Mucociliary Velocity and Clearance of Streptococcus pneumoniae. Am J Respir Cell Mol Biol 2010; 42 (4): 450-60.

60. Wilson R, Dowling RB, Jackson AD. The biology of bacterial colonization and invasion of the respiratory mucosa. Eur Respir J 1996; 9: 1523-30.

61. Blanco JL, García ME. Immune response to fungal infections. Vet Immunol Immunopathol 2008; 125: 47-70.

62. Bartlett JA, Fischer AJ, Mccray PB Jr. Innate immune functions of the airway epithelium. Contrib Microbiol 2008; 15: 147-63.

63. Lee ChJ, Lee HJ, Lee SY, Cho K, Jeon B, Lee JW, et al. Effect of Ambroxol on Secretion, Production and Gene Expression of Mucin from Cultured Airway Epithelial Cells. Biomol Ther 2011; 19 (1): 65-9.

64. Rogers DF. Mucoactive Agents for Airway Mucus Hypersecretory Diseases. Respir Care 2007; 52 (9): 1176-97.

65. Baraniuk JN, Zheng Y. Treatment of mucous hypersecretion. Clin Expe Allergy Rev 2010; 10: 12-9.

66. Rubin BK. Mucolytics, expectorants, and mucokinetic medications. Respir Care 2007; 52 (7): 859-65.

67. Wallis C. Mucolytic therapy in cystic fibrosis. J R Soc Med 2001; 94: 17-24.

68. Decramer M, Rutten-van Mölken M, Dekhuijzen PN, Troosters T, van Herwaarden C, Pellegrino R, et al. Effects of $\mathrm{N}$-acetylcysteine on outcomes in chronic obstructive pulmonary disease (Bronchitis Randomized on NAC Cost-Utility Study, BRONCUS): a randomised 
placebo-controlled trial. Lancet 2005; 365 (9470): 155260.

69. van Overveld FJ, Demkow U, Górecka D, de Backer WA, Zielinski J. New developments in the treatment of COPD: comparing the effects of inhaled corticosteroids and N-acetylcysteine. J Physiol Pharmacol 2005; 56 Suppl 4: 135-42.

70. Black PN, Morgan-Day A, McMillan TE, Poole PJ, Young RP. Randomised, controlled trial of N-acetylcysteine for treatment of acute exacerbations of chronic obstructive pulmonary disease [ISRCTN21676344]. BMC Pulm Med 2004; 4: 13.

71. Anzueto A, Jubran A, Ohar JA, Piquette CA, Rennard SI, Colice G, et al. Effects of aerosolized surfactant in patients with stable chronic bronchitis: a prospective randomized controlled trial. J Am Med Assoc 1997; 278 (17): 1426-31.

72. Guleria R, Singh TR, Sinha S, Padhy K, Gupta K, Pande JN. Effect of single inhalation of a salbutamol, ipratropium bromide and beclomethasone on mucociliary clearance in patients with chronic obstructive airway disease. Indian J Chest Dis Allied Sci 2003; 45 (4): 241-6.

73. Meyer T, Reitmeir P, Brand P, Herpich C, Sommerer K, Schulze A, et al. Effects of formoterol and tiotropium bromide on mucus clearance in patients with COPD. Respir Med 2011; 105 (6): 900-6.

74. Restrepo RD. Inhaled adrenergics and anticholinergics in obstructive lung disease: Do they enhance mucoci- liary clearance? Respir Care 2007; 52 (9): 1159-75.

75. Piatti G, Ambrosetti U, Santusc P, Allegra L. Effects of salmeterol on cilia and mucus in COPD and pneumonia patients. Pharmacol Res 2005; 51 (2): 165-8.

76. Maneechotesuwan K, Essilfie-Quaye S, Meah S, Kelly C, Kharitonov SA, Adcock IM, et al. Formoterol attenuates neutrophilic airway inflammation in asthma. Chest 2005; 128 (4): 1936-42.

77. Hasani A, Toms N, Agnew JE, Sarno M, Harrison AJ, Dilworth P. The effect of inhaled tiotropium bromide on lung mucociliary clearance in patients with COPD. Chest 2004; 125 (5): 1726-34.

78. Ballmann M, Hardt HVD. Hypertonic saline and recombinant human DNase: a randomised cross-over pilot study in patients with cystic Fibrosis. J Cyst Fibros 2002; 1 (1): 35-7.

79. Suri R, Grieve R, Normand C, Metcalfe C, Thompson $\mathrm{S}$, Wallis C, et al. Effects of hypertonic saline, alternate day and daily rhDNase on healthcare use, costs and outcomes in children with cystic fibrosis. Thorax 2002; 57 (10): 841-6.

80. Barbato A, Frischer T, Kuehni CE, Snijders D, Azevedo I, Baktai G, et al. Primary ciliary dyskinesia: a consensus statement on diagnostic and treatment approaches in children. Eur Respir J 2009; 34: 1264-76.

81. Strieter RM, Belperio JA, Keane MP. Cytokines in innate host defense in the lung. J Clin Invest 2002; 109: 699705 . 